\title{
Evaluation of the risk of fracture in type 2 diabetes mellitus patients with incretins: an updated meta-analysis
}

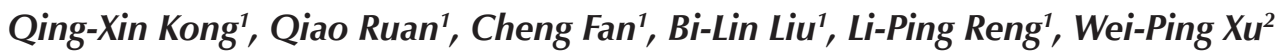 \\ ${ }^{1}$ Chongqing Chemical Industry Vocational College, Chongqing, China \\ ${ }^{2}$ Hospital of the Southwest University of Political Science and Law, Chongqing, China
}

\begin{abstract}
Introduction: The effect of incretins including dipeptidyl peptidase 4 inhibitors (DPP4-Is) and glucagon-like peptide 1 receptor agonists (GLP1-ras) in the treatment of type 2 diabetes increasing the risk of fracture remains controversial. No meta-analysis has been written to discuss this from the prospective interventional studies. The objective was to evaluate the association between the use of incretins and fracture risk.

Material and methods: Multiple databases were searched for original articles that investigated the relationship between the use of incretin agents and fracture risk, up to December 2019. Trials using the Mantel-Haenszel method to calculate OR and 95\% CI were pooled. The multiple sensitivity, heterogeneity, publication bias, and quality were analysed among the studies to evaluate the robustness of results. Results: The fixed-effects model was used on account of the $\mathrm{I}^{2}$ test for heterogeneity $\left(\mathrm{I}^{2}=0.0 \%\right)$. Incretins were not associated with fracture risk [0.97 (95\% CI: 0.88-1.08)]. But in the subgroup analysis, when sitagliptin 100 mg per day (OR 0.495, 95\% CI: 0.304-0.806) or liraglutide $1.8 \mathrm{mg}$ per day was administered (OR $0.621,95 \%$ CI: $0.413-0.933$ ), it reduced fracture risk. The sensitivity analysis and publication bias prompted the robustness of results.

Conclusions: This meta-analysis suggested that the current use of incretins not only is safe for fracture in type 2 diabetes patients from RCT studies, but also, when sitagliptin $100 \mathrm{mg}$ or liraglutide $1.8 \mathrm{mg}$ per day was administered, it may exhibit protective effects on bone metabolism. (Endokrynol Pol 2021; 72 (4): 319-328)
\end{abstract}

Key words: incretins; GLP-1; DPP-4; fracture; meta-analysis; RCT

\section{Introduction}

Fractures are associated with increased morbidity and mortality, and result in a considerable economic burden upon health care systems [1]. Although bone fragility and fractures have not traditionally been considered as major complications of type 2 diabetes mellitus, patients with type 2 diabetes mellitus (T2DM) are at higher risk of bone fragility and fractures [2] in terms of disease pathology and associated hypoglycaemic drug therapy [3]. For example, thiazolidinediones (TZD) have a worldwide known adverse effect of increasing the fracture risk in postmenopausal women [4], which is recognized by doctors [5]. With the increase of age and the progress of the disease, the probability of osteoporosis in patients with type 2 diabetes will increase. Therefore, we believe that it is necessary for individuals to carry out risk assessment when choosing hypoglycaemic drugs.

As a new class of hypoglycaemic drugs, incretins are used increasingly in the clinic, especially for the elderly, because of their efficacy and safety due to their unique pharmacological action [6]. Incretins are a group of hormones that are synthesized and secreted by gut endo- crine cells under the stimulus of food nutrients, which promotes insulin secretion and inhibits inappropriate secretion of glucagon of alpha cells to affect the persons' appetite and hypoglycaemic effect [7]. Incretins mainly include glucose-dependent insulinotropic polypeptide (GIP) and glucagon-like peptide 1 (GLP-1), which are rapidly metabolized by dipeptidyl peptidase 4 (DPP-4) in the body. The drugs are developed based on the principle of incretins, which mainly include glucagon-like peptide-1 receptor agonists (GLP1-ras) and dipeptidyl peptidase-4 inhibitors (DPP4-Is). GLP1-ras have a significantly extended GLP-1 half-life because they are not recognized by DPP-4 enzyme. And DPP4-Is can improve the GLP-1 level in the body by inhibiting DPP-4 enzyme. As a result, DPP4-Is and GLP1-ras play a role by enhancing GLP-1 levels in the body [8].

The relationship between incretins and fracture is relatively rarely described, and data is mostly derived from meta-analyses. Most previous meta-analyses [9-14] tend to take either GLP1-ras or DPP4-Is as an object. The mechanism of action for both GLP1-ras and DPP4-Is are similar in regard to GLP-1 level enhancement in the body. What is more, we hope to evaluate 
whether the class of drugs increase the risk of fracture in the treatment of type 2 diabetes by a larger sample size, so it is reasonable to take incretins as the clarification.

As the only study to take the incretins as the object, Driessen et al. [15] conducted a meta-analysis that only included four studies by searching Pubmed and Embase. On one hand, those four retrospective studies were from the same author, which may cause selection bias and reporting bias. On the other hand, the validity and completeness of the data in the retrospective primary care database will affect the correctness of the analysis. These may cause attrition bias. The RCT, which has the highest level of evidence for quality grades, effectively reduces or eliminates the imbalance between the treatment and control groups. Therefore, we first used a pool of RCT data by searching more databases to conduct an updated meta-analysis, in order to find strong evidence about the relationship between the use of incretins and the risk of fracture.

\section{Material and methods}

The updated meta-analysis was conducted in accordance with the PRISMA (Preferred Reporting Items for Systematic Reviews and Meta-Analyses) statement [16], and the protocol was registered at PROSPERO (registration number: CRD42018102261).

\section{Data Sources and Searches}

A systematic and comprehensive search of Pubmed, Embase, Web of Science, and the Cochrane Library was performed by three investigators (QXK, QR, and CF). Data were collected on all RCTs in humans up to December 2019. The language of the articles was limited to English. Differences in abstracted data between the reviewers were resolved by other reviewers (BLL and LPR). We combined both specific subject headings (e.g. MeSH terms) and free text terms to identify all of the potentially relevant articles in the databases. Considering that there were many articles about the efficacy and safety of drugs, it seemed inappropriate to limit the fracture as a search term. As a result, the search terms were used as follows: (1) "DPP-4", "dipeptidyl peptidase 4", "alogliptin", "linagliptin", "saxagliptin", "sitagliptin", "vildagliptin", "glucagon like peptide 1", "glucagon-like peptide-1", “GLP-1", “GLP 1", "liraglutide", "exenatide", "lixisenatide", "albiglutide", "dulaglutide", "semaglutide"; (2) "randomized", "placebo", and "randomized controlled trial". The trials that were completed but unpublished were identified through a search on the www.clinicaltrials.gov website, and fracture data from eligible trials were confirmed. In addition, we also manually searched the reference lists of the related studies as far as possible to identify any additional studies.

\section{Study selection}

The trials were included if they satisfied all of the following criteria: (1) randomized clinical trials in type 2 diabetes patients; (2) duration of at least 12 weeks; (3) comparing incretins with placebo or active drugs; and (4) data on bone fracture available. Trials with incomplete original data or with 2 zero events were excluded from the analysis. If several studies with the same population were retrieved, the one with the most complete data was used.

\section{Data extraction}

Three reviewers (QXK, QR, and $\mathrm{CF}$ ) identified studies and independently abstracted data according to the predefined protocol. Any resulting discrepancies of the study selection process were resolved by discussion with other reviewers (BLL and LPR). The following information was extracted independently from eligible RCTs using a standardized form: trial characteristics (author's name, year of publication, journal, National Clinical Trial number, study design, type and dose of incretins and the comparison drugs, sample size of the treatment and control groups, length of follow-up), and participants' baseline (mean age, mean $\mathrm{HbA}_{1{ }^{\prime}}$ mean $\mathrm{BMI}$, mean duration of diabetes) and fracture outcomes (number of fracture events per group, type of fracture).

\section{Quality assessment}

Three independent reviewers (QXK, QR, and CF) assessed the quality of the involved RCTs using the Cochrane Collaboration's risk of bias tool. Quality is only seen for descriptive purposes, but not as the criterion for the selection of trials. The bias evaluation was based on all of the following seven domains: (1) random sequence generation (selection bias); (2) allocation concealment (selection bias); (3) blinding of participants and personnel (performance bias); (4) blinding of outcome assessment (detection bias); (5) incomplete outcome data (attrition bias); (6) selective reporting (reporting bias); and (7) other biases. For each domain, the risk of bias was divided into low, high, and ambiguous. Details for assessment of risk of bias are presented in Figure 1 and 2.

\section{Data analysis}

Trials using the Mantel-Haenszel method were pooled to calculate OR and $95 \%$ CIs. $p$ values $<0.05$ were considered statistically significant. The result of the Cochrane's $Q$ test is the key to choosing the fixed or random effects model. If $\mathrm{I}^{2}<50 \%$, a fixed effects model was used, suggesting that there was no significant heterogeneity; otherwise, a random effects model was applied [17].

Heterogeneity evaluation was performed by the Galbraith plot, multiple meta-regression, and subgroup analyses. Pre-defined subgroup analyses were performed for trials that included different areas (Asian vs. non-Asian); different classifications of incretins (DPP4-Is vs. GLP1-ras); different types of incretins (alogliptin, linagliptin, saxagliptin, sitagliptin, anagliptin, and vildagliptin); different doses of incretins [saxagliptin $(2.5 \mathrm{mg}, 5 \mathrm{mg}, 10 \mathrm{mg})$, sitagliptin $(25 \mathrm{mg}, 50 \mathrm{mg}, 100 \mathrm{mg})$, vildagliptin $(50 \mathrm{mg}, 100 \mathrm{mg})$, alogliptin $(12.5 \mathrm{mg}$, $25 \mathrm{mg})$, linagliptin $(2.5 \mathrm{mg}, 5 \mathrm{mg})$, anagliptin $200 \mathrm{mg}$, omarigliptin $25 \mathrm{mg}$, dulaglutide $(0.75 \mathrm{mg}, 1.5 \mathrm{mg})$, exenatide ( $30 \mu \mathrm{g}, 20 \mu \mathrm{g})$, liraglutide $(0.9 \mathrm{mg}, 1.2 \mathrm{mg}, 1.8 \mathrm{mg}, 3.0 \mathrm{mg})$, lixisenatide $20 \mu \mathrm{g}$, albiglutide $30 \mathrm{mg}$ ]; different types of control (active drug vs. placebo drug); different lengths of follow-up (less than 52 weeks vs. 52 weeks or more); mean age (less than 60 years vs. 60 years or more); mean $\mathrm{HbA}_{1 \mathrm{c}}$ (less than $8 \%$ vs. $8 \%$ or more); mean BMI (less than $32 \mathrm{~kg} / \mathrm{m}^{2} v s .32 \mathrm{~kg} / \mathrm{m}^{2}$ or more); or mean diabetes duration (less than 7 years $v s .7$ years or more).

Sensitivity analysis was performed to evaluate the robustness of the results by using different statistical models (fixed-effect model vs. random-effect model) and different effect measures [odds ratio $(\mathrm{OR})$ vs. relative risk (RR)]. We also undertook the nonparametric "trim and fill" procedure and excluded trials with only event to further assess the robustness in this meta-analysis. Finally, publication bias was investigated graphically with funnel plots. The asymmetry of the funnel plots was evaluated by Egger's tests $[18,19]$ with $p$ values $<0.10$ suggesting the presence of small study effects [20, 21]. All statistical analyses were performed with Revman (Version 5.3) and Stata (Version 14.0).

\section{Results}

\section{Search results}

There were 4651 unique titles and abstracts identified through a search of the electronic databases and the www.clinicaltrials.gov website. After excluding du- 


\begin{tabular}{|c|c|c|c|c|c|c|c|c|c|c|c|c|c|c|c|c|c|c|c|c|c|c|c|}
\hline 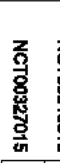 & & & & & & & & & & & & & & & & & & & & & $\begin{array}{l}\frac{8}{2} \\
\frac{8}{2}\end{array}$ & & \\
\hline$\rightarrow$ & 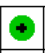 & • & 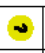 & 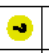 & 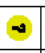 & $\sim$ & 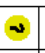 & • & • & $\oplus$ & ๑) & $\approx$ & - & & 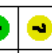 & 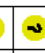 & $\bullet$ & $\epsilon$ & 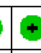 & $\oplus$ & $\odot$ & 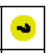 & Random sequences generastlon (solection blas) \\
\hline - & 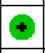 & • & $\bullet$ & $\oplus$ & 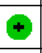 & • & • & $\bullet$ & ๑ & $\oplus$ & $\oplus$ & - & 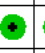 & $\rightarrow$ & 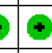 & $\bullet$ & $\bullet$ & $\rightarrow$ & $\infty$ & $\varphi$ & ( & - & Allocation concosalment (sallection blas) \\
\hline$\oplus$ & + & $\oplus$ & + & $\oplus$ & + & 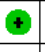 & $\oplus$ & ( & $\oplus$ & + & ( & $\oplus$ & ๑ & + & + & 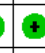 & $\oplus$ & $\omega$ & 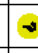 & + & $\oplus$ & $\oplus$ & 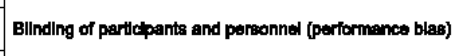 \\
\hline+ & + & 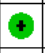 & $\omega$ & $\bullet$ & + & (†) & † & $(4$ & ( & + & ( & † & + & $\omega$ & $\bullet-$ & + & $\omega$ & $\omega$ & 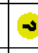 & 4 & + & (†) & Blinding of outbome assossmment (dothection biess) \\
\hline$\oplus$ & ๑ & $\bullet$ & • & 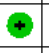 & $\oplus$ & (•) & • & ( & ๑ & - & • & 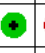 & (a) & 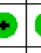 & 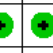 & 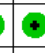 & $\odot$ & $\epsilon$ & + & $\omega$ & 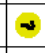 & 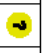 & 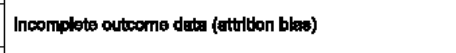 \\
\hline$\oplus$ & ๑ & • & • & $\odot$ & $\oplus$ & • & • & • & ๑ & ๑ & $\oplus$ & • & $\rightarrow$ & 5 & 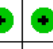 & 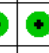 & 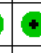 & $\sim$ & + & 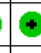 & 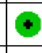 & • & Selective reporthng (roporting blas) \\
\hline$\oplus$ & $\oplus$ & $\rightarrow$ & & & $\oplus$ & ๑ & $\bullet$ & ๑ & ๑ & ๑ & ๑ & ๑ & $\bullet$ & & 4 & + & $\omega$ & $\epsilon$ & $\infty$ & & & $\bullet$ & Other blas \\
\hline & & & & & & & & & & & & & & & & & & & & & & 总 & \\
\hline ( & ( & $(4$ & $\sim$ & (4) & ( ) & ( & $\rightarrow$ & + & $\sim$ & + & $\rightarrow$ & $\bullet$ & + & 6 & + & $\sim$ & + & + & + & $\sim$ & $\oplus$ & + & 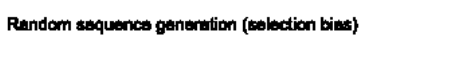 \\
\hline (†) & (†) & $(4$ & $\rightarrow$ & (4) & ( & ( & ( & + & ( & + & + & 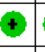 & $\oplus$ & & \pm & 4 & + & + & + & $\sim$ & + & + & Allocertion concosalmont (soloction biess) \\
\hline (†) & (†) & (†) & $\rightarrow$ & (†) & (†) & ( & ( & ๑ & ( & ( & 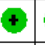 & ( & $\oplus$ & 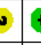 & + & 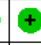 & + & \pm & + & & + & $\oplus$ & Blinding of participante and personnel (pertiomenosos bisas) \\
\hline$\rightarrow$ & (†) & (†) & $\rightarrow$ & $($ ๑ & (†) & ( & $\rightarrow$ & ๑ & $\sim$ & ( & ( & ( & $(4$ & & $\sim$ & $\omega$ & $\sim$ & $\oplus$ & $\oplus$ & $\omega$ & + & $\sim$ & 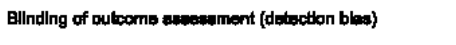 \\
\hline (†) & ๑ & (†) & (†) & (†) & (†) & $\rightarrow$ & ( & ๑ & ๑ & ( & $\sim$ & (七) & $\rightarrow$ & 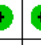 & + & $\omega$ & 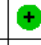 & \pm & 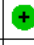 & $\oplus$ & $\oplus$ & $\oplus$ & 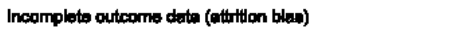 \\
\hline (•) & (†) & $\bullet$ & ๑ & • & ๑ & • & • & • & $\sim$ & • & $\bullet$ & $\bullet$ & + & 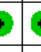 & 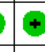 & + & + & + & + & + & + & • & Solectve reporthg (roporting bless) \\
\hline • & 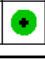 & • & • & $\bullet$ & - & - & • & • & $\bullet$ & $\bullet$ & - & $\bullet$ & 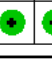 & . & + & + & 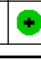 & 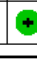 & $\epsilon$ & 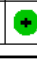 & $\omega$ & () & Other blem \\
\hline & & & & & & & & & & & & & & & & & & 蒿 & 8 & 荡 & 跑 & 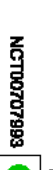 & \\
\hline$\bullet$ & $\rightarrow$ & $\oplus$ & $\oplus$ & $\oplus$ & $\oplus$ & $\sim$ & $\rightarrow$ & 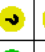 & 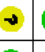 & $\oplus$ & $\oplus$ & $\rightarrow$ & +4 & 5 & $\oplus$ & $\hookrightarrow$ & 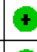 & $\oplus$ & 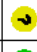 & 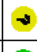 & $\omega$ & ( & 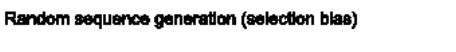 \\
\hline ๑ & ( & 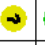 & + & + & $\sim$ & $\oplus$ & $\oplus$ & ( & + & + & ๑ & ๑) & Ð & + & \pm & \pm & 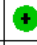 & 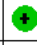 & $\oplus$ & $\oplus$ & $\oplus$ & ๑) & Alocation concesalmant (selection biest) \\
\hline+ & ( & $\sim$ & $\oplus$ & + & $\sim$ & + & + & $(+$ & + & \pm & \pm & 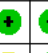 & + & + & + & + & 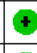 & 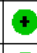 & $\oplus$ & + & + & $($ ๑) & 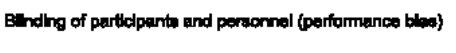 \\
\hline$\sim$ & $\rightarrow$ & $\bullet$ & $\bullet$ & $\bullet$ & $\sim$ & 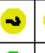 & $\bullet$ & $\sim$ & $\sim$ & $\oplus$ & e. & $\rightarrow$ & 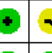 & +4 & 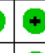 & $\bullet$ & $\bullet$ & $\oplus$ & $\sim$ & $\sim$ & 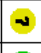 & • & 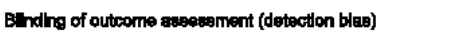 \\
\hline$\bullet$ & $\bullet$ & $\bullet$ & $\bullet$ & $\oplus$ & $\oplus$ & $\bullet$ & $\bullet$ & $\bullet$ & + & $\oplus$ & $\rightarrow$ & • & +4 & ? & + & $\bullet$ & $\bullet$ & $\oplus$ & 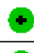 & $\oplus$ & $\oplus$ & $\bullet$ & Incompleba outcome deta (attitlion blas) \\
\hline$\bullet$ & • & • & • & $\bullet$ & $\bullet$ & $\bullet$ & $\bullet$ & - & $\bullet$ & $\oplus$ & 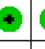 & • & ๑ & $=$ & 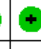 & $\bullet$ & $\bullet$ & $\bullet$ & $\odot$ & + & 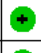 & $\bullet$ & Solective reporting (reporttng blas) \\
\hline 4 & $\sim$ & $\sim$ & + & + & $\oplus$ & $(+$ & $\oplus$ & + & + & + & $\oplus$ & +5 & +5 & (5 & 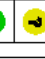 & + & 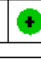 & 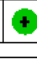 & $\oplus$ & $\oplus$ & + & $\oplus$ & Other blas \\
\hline & & & & & & & & & & & & & & & & & & & & & & ב & \\
\hline 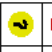 & $\bullet$ & $\oplus$ & 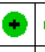 & • & 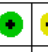 & $\bullet$ & - 6 & +5 & 4. & + & $\bullet$ & 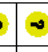 & - & • & - & 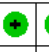 & $\oplus$ & 2. & 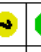 & $\rightarrow$ & e 4 & $\bullet$ & Random soquencos generartion (schection blas) \\
\hline$\bullet$ & $\bullet$ & $\oplus$ & 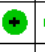 & $\bullet$ & $\rightarrow$ & 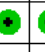 & *6 6 & $\rightarrow$ & +6 & + & $\rightarrow$ & $\infty$ & $\bullet$ & • & • & & + & $\rightarrow$ & $\rightarrow$ & +3 & + & $\bullet$ & Allocation concosedment (selection blas) \\
\hline (4) & (4) & (†) & + & $\oplus$ & $\sim$ & $\oplus$ & +6 & +4 & 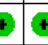 & 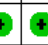 & $\omega$ & + & $\oplus$ & 9 & (†) & & $\oplus$ & $\sim$ & $\sim$ & +6 & $+屯$ & 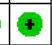 & 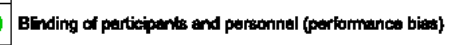 \\
\hline$\oplus$ & $\bullet$ & $\sim$ & $\sim$ & 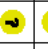 & $\sim 5$ & $\rightarrow$ & +6 & +5 & $0-5$ & 04 & $\omega$ & $\omega$ & $\oplus$ & $\oplus$ & 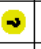 & $\sim$ & $\rightarrow$ & $\rightarrow$ & $\rightarrow$ & & & 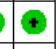 & 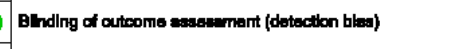 \\
\hline - & ๑ & ๑ & 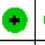 & ๑) & * & e & $\rightarrow$ & क & (c) & 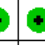 & 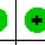 & $\epsilon$ & $\bullet$ & $\odot$ & • & $\oplus$ & 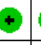 & $\rightarrow$ & • & $\rightarrow$ & & $\bullet$ & 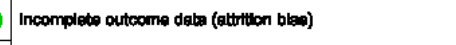 \\
\hline$\bullet$ & • & $\bullet$ & $\bullet$ & - & ๑) & • & * & c) & (c) & 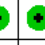 & 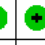 & 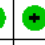 & - & • & • & $\bullet$ & • & • & • & $\rightarrow$ & & $\bullet$ & Selective reporting (roporttno blas) \\
\hline$\oplus$ & (屯) & $\oplus$ & $\oplus$ & $\oplus$ & $\oplus$ & • & +6 & t) & t. & 4 & \pm & + & 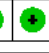 & + & ๑ & $\oplus$ & $\oplus$ & † & 5 & & & $\oplus$ & Other blas \\
\hline & & & & & & & & & & & & & & & & & & & & & & & \\
\hline$\bullet$ & • & $\rightarrow$ & $\bullet$ & $\bullet$ & $\rightarrow$ & $\rightarrow$ & +- & 0 & +5 & 1 & $\div$ & $\bullet$ & $\bullet$ & $\oplus$ & ( ) & $\bullet$ & $\bullet$ & $\rightarrow$ & $\rightarrow$ & 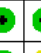 & 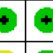 & + & 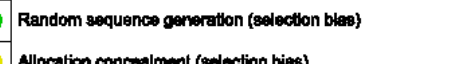 \\
\hline ๑) & ๑) & 4 & $\rightarrow$ & $\oplus$ & $\sim$ & 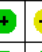 & $\rightarrow$ & 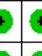 & 5 & + & 4 & $\odot$ & 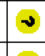 & $\rightarrow$ & $\sim$ & 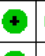 & + & $\rightarrow$ & +5 & 5 & 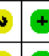 & $\rightarrow$ & Nlocation concosalment (solection biess) \\
\hline ๑ & ๑ & $\oplus$ & $\sim$ & $\oplus$ & a & \pm & + & & & + & 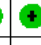 & $\sim$ & $\omega$ & $\omega$ & $\sim$ & 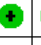 & † & + & +5 & + & 0 & $\sim$ & 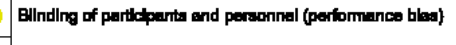 \\
\hline$\sim$ & (•) & $\omega$ & $\sim$ & 2 & a. & $\rightarrow$ & + & & ( & $\omega$ & 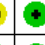 & $\sim$ & 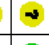 & $\omega$ & $\sim$ & $\bullet$ & $\rightarrow$ & + & - & c 5 & 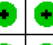 & $\sim$ & 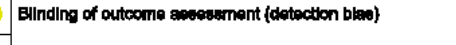 \\
\hline$\omega$ & $\bullet$ & $\bullet$ & • & • & • & $\rightarrow$ & $\rightarrow$ & c & 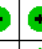 & + & 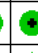 & $\epsilon$ & $\bullet$ & + & $\bullet$ & ( & 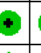 & + & + & & +4 & $\bullet$ & 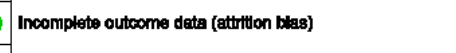 \\
\hline ๑) & ๑ & + & + & + & 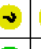 & $\rightarrow$ & +5 & & +4 & 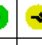 & 4 & 4 & $\oplus$ & $\oplus$ & () & $\sim$ & $\oplus$ & $\rightarrow$ & + & & +5 & + & Salective reporting (roporting bias) \\
\hline † & & & & & & & & & & & & & $\oplus$ & & & & & & & & & & Other blea \\
\hline
\end{tabular}

Figure 1. "Risk of bias" graph: review authors' judgements about each risk of bias item presented as percentages across all included studies 


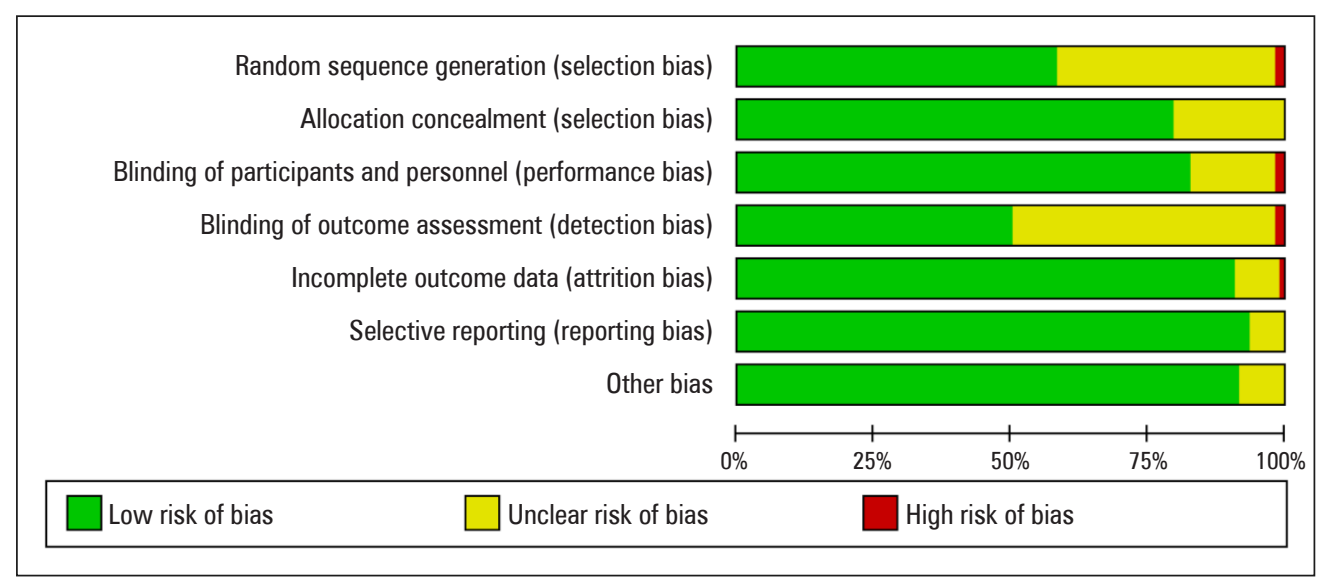

Figure 2. "Risk of bias" summary: review authors' judgements about each risk of bias item for each included study

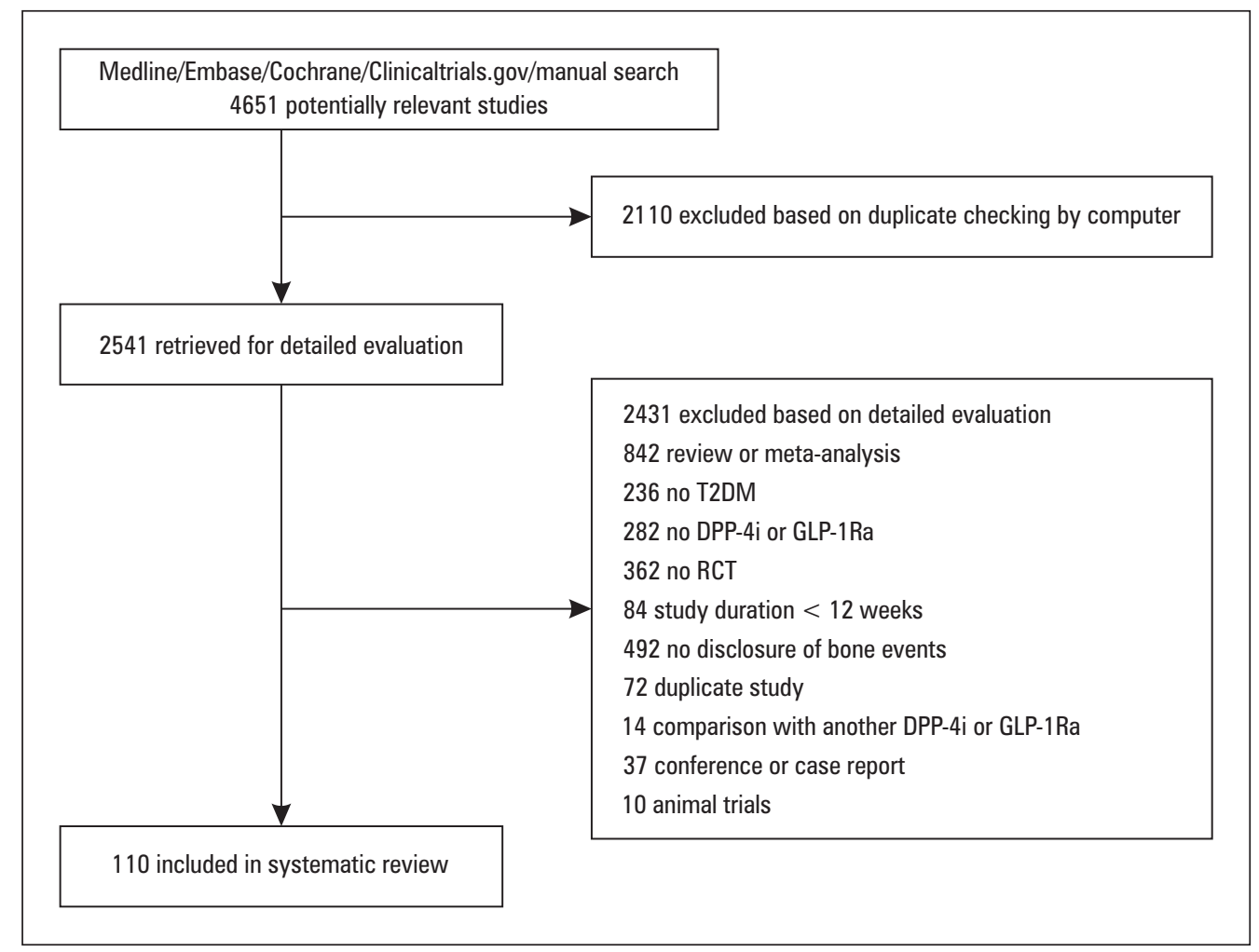

Figure 3. Study selection process; T2DM - diabetes mellitus; DPP-4 - dipeptidyl peptidase 4; GLP - glucagon-like peptide; $R C T$ - randomized control trial

plicate publications and screening titles and abstracts, we retrieved 2541 reports for full text evaluation. As a result, 110 randomized control trials (RCTs), including 74 from electronic database and 36 from the trial registry (available from https://clinicaltrials.gov) met the inclusion criteria. The details of the study selection flow are summarized in Figure 1.

\section{Study characteristics}

The baseline characteristics of trials are listed in Table 1. Out of these 110 RCTs, a total of 12 drug subjects were included, of which NCT00295633, NCT00294723, NCT00856284, NCT00121667, NCT00722371, NCT01272219, NCT00103857, NCT01075282, NCT01191268, and NCT01621178 were compared with different doses. Among them, NCT00121667 and NCT00722371 made a comparison of three doses, and the rest made a comparison of two doses. Therefore, 122 trials were included in this meta-analysis, with 96 double-blind trials. The final sample consisted of 111,539 individuals (58,706 individuals were in the experimental group and 52,833 individuals were in the control group), 
Table 1. Subgroup analyses of changes

\begin{tabular}{|c|c|c|c|c|c|c|c|}
\hline & Group & $\begin{array}{l}\text { Number } \\
\text { of study }\end{array}$ & WMD & $\begin{array}{c}\text { Net change } \\
(95 \% \mathrm{CI})\end{array}$ & $\mathrm{p}$-value & $\mathbf{P}_{\text {heterogeneity }}$ & $1^{2}$ \\
\hline \multirow{12}{*}{ DPP-4/GLP-1 } & Vildagliptin & 6 & 0.859 & $0.287-2.566$ & 0.785 & 0.656 & $0.0 \%$ \\
\hline & Sitagliptin & 33 & 0.913 & $0.758-1.100$ & 0.340 & 0.969 & $0.0 \%$ \\
\hline & Exenatide & 9 & 1.872 & $0.901-3.892$ & 0.093 & 0.817 & $0.0 \%$ \\
\hline & Saxagliptin & 14 & 0.995 & $0.838-1.182$ & 0.956 & 0.357 & $0.0 \%$ \\
\hline & Alogliptin & 8 & 0.807 & $0.562-1.158$ & 0.244 & 0.929 & $0.0 \%$ \\
\hline & Liraglutide & 11 & 0.714 & $0.499-1.023$ & 0.067 & 0.483 & $0.0 \%$ \\
\hline & Linagliptin & 17 & 1.408 & $0.859-2.306$ & 0.175 & 0.965 & $0.0 \%$ \\
\hline & Lixisenatide & 8 & 1.154 & $0.511-2.606$ & 0.730 & 0.903 & $0.0 \%$ \\
\hline & Albiglutide & 2 & 2.668 & $0.291-24.490$ & 0.386 & 0.918 & $0.0 \%$ \\
\hline & Dulaglutide & 10 & 1.051 & $0.532-2.078$ & 0.886 & 0.589 & $0.0 \%$ \\
\hline & Omarigliptin & 3 & 1.509 & $0.765-2.973$ & 0.235 & 0.620 & $0.0 \%$ \\
\hline & Anagliptin & 1 & 8.217 & $0.410-164.684$ & 0.168 & 0.000 & $0.0 \%$ \\
\hline \multirow{2}{*}{ Incretins } & DPP-4 & 82 & 0.977 & $0.873-1.095$ & 0.692 & 0.993 & $0.0 \%$ \\
\hline & GLP-1 & 40 & 0.944 & $0.722-1.234$ & 0.672 & 0.835 & $0.0 \%$ \\
\hline \multirow{2}{*}{ Comparator } & Placebo & 63 & 0.973 & $0.870-1.089$ & 0.638 & 0.925 & $0.0 \%$ \\
\hline & Active drug & 59 & 0.966 & $0.734-1.272$ & 0.806 & 0.985 & $0.0 \%$ \\
\hline \multirow{2}{*}{ Region } & Asian & 17 & 1.105 & $0.609-2.004$ & 0.743 & 0.736 & $0.0 \%$ \\
\hline & Non-Asian & 58 & 0.990 & $0.859-1.156$ & 0.894 & 0.943 & $0.0 \%$ \\
\hline \multirow{2}{*}{ Age [y] } & $\geq 60$ & 24 & 0.954 & $0.848-1.073$ & 0.432 & 0.576 & $0.0 \%$ \\
\hline & $<60$ & 93 & 1.015 & $0.808-1.276$ & 0.896 & 0.995 & $0.0 \%$ \\
\hline \multirow{2}{*}{ BMI $\left[\mathrm{kg} / \mathrm{m}^{2}\right]$} & $\geq 32$ & 24 & 0.976 & $0.647-1.473$ & 0.909 & 0.747 & $0.0 \%$ \\
\hline & $<32$ & 55 & 0.947 & $0.819-1.095$ & 0.458 & 0.984 & $0.0 \%$ \\
\hline \multirow{2}{*}{ Length of follow-up [y] } & $\geq 52$ & 59 & 0.967 & $0.865-1.080$ & 0.551 & 0.875 & $0.0 \%$ \\
\hline & $<52$ & 63 & 1.014 & $0.748-1.376$ & 0.928 & 0.993 & $0.0 \%$ \\
\hline \multirow{2}{*}{ Duration [y] } & $\geq 7$ & 30 & 0.960 & $0.849-1.087$ & 0.523 & 0.968 & $0.0 \%$ \\
\hline & $<7$ & 29 & 0.819 & $0.565-1.188$ & 0.294 & 0.767 & $0.0 \%$ \\
\hline \multirow{2}{*}{$\mathrm{HbA}_{1 \mathrm{c}}(\%)$} & $\geq 8$ & 58 & 0.896 & $0.777-1.034$ & 0.132 & 0.972 & $0.0 \%$ \\
\hline & $<8$ & 29 & 1.060 & $0.885-1.270$ & 0.529 & 0.909 & $0.0 \%$ \\
\hline \multirow{15}{*}{ Dose } & Albiglutide $30 \mathrm{mg}$ & 2 & 2.668 & $0.291-24.490$ & 0.386 & 0.918 & $0.0 \%$ \\
\hline & Alogliptin $12.5 \mathrm{mg}$ & 1 & 0.744 & $0.166-3.334$ & 0.699 & 0.000 & $0.0 \%$ \\
\hline & Alogliptin $25 \mathrm{mg}$ & 65 & 0.994 & $0.458-2.157$ & 0.989 & 0.838 & $0.0 \%$ \\
\hline & Anagliptin $200 \mathrm{mg}$ & 1 & 8.217 & $0.410-164.684$ & 0168 & 0.000 & $0.0 \%$ \\
\hline & Dulaglutide $0.75 \mathrm{mg}$ & 4 & 0.869 & $0.302-2.498$ & 0.794 & 0.464 & $0.0 \%$ \\
\hline & Dulaglutide $1.5 \mathrm{mg}$ & 6 & 1.206 & $0.491-2.962$ & 0.684 & 0.423 & $0.0 \%$ \\
\hline & Exenatide 30 ug & 1 & 3.000 & $0.122-74.025$ & 0.502 & 0.000 & $0.0 \%$ \\
\hline & Exenatide 20 ug & 7 & 1.662 & $0.759-3.639$ & 0.204 & 0.703 & $0.0 \%$ \\
\hline & Linagliptin $2.5 \mathrm{mg}$ & 2 & 0.881 & $0.123-6.294$ & 0.900 & 0.877 & $0.0 \%$ \\
\hline & Linagliptin 5 mg & 15 & 1.452 & $0.871-2.420$ & 0.153 & 0.929 & $0.0 \%$ \\
\hline & Liraglutide $0.9 \mathrm{mg}$ & 1 & 0.166 & $0.007-4.096$ & 0.272 & 0.000 & $0.0 \%$ \\
\hline & Liraglutide $1.2 \mathrm{mg}$ & 2 & 0.552 & $0.117-2.606$ & 0.453 & 0.170 & $47.0 \%$ \\
\hline & Liraglutide $1.8 \mathrm{mg}$ & 4 & 0.621 & $0.413-0.933$ & 0.022 & 0.748 & $0.0 \%$ \\
\hline & Liraglutide $3.0 \mathrm{mg}$ & 3 & 3.964 & $0.885-17.753$ & 0.072 & 0.983 & $0.0 \%$ \\
\hline & Lixisenatide 20 ug & 8 & 1.154 & $0.511-2.606$ & 0.730 & 0.903 & $0.0 \%$ \\
\hline
\end{tabular}


Table 1. Subgroup analyses of changes

\begin{tabular}{|c|c|c|c|c|c|c|c|}
\hline & Group & $\begin{array}{l}\text { Number } \\
\text { of study }\end{array}$ & WMD & $\begin{array}{l}\text { Net change } \\
(95 \% \mathrm{Cl})\end{array}$ & $\mathrm{p}$-value & $\mathbf{P}_{\text {heterogeneity }}$ & $I^{2}$ \\
\hline \multirow{9}{*}{ Dose } & Omarigliptin $25 \mathrm{mg}$ & 2 & 1.453 & $0.724-2.914$ & 0.293 & 0.387 & $0.0 \%$ \\
\hline & Saxagliptin 10 mg & 2 & 3.329 & $0.376-29.459$ & 0.280 & 0.933 & $0.0 \%$ \\
\hline & Saxagliptin $2.5 \mathrm{mg}$ & 4 & 1.844 & $0.584-5.821$ & 0.297 & 0.662 & $0.0 \%$ \\
\hline & Saxagliptin 5 mg & 8 & 0.970 & $0.814-1.155$ & 0.729 & 0.159 & $33.7 \%$ \\
\hline & Sitagliptin 100 mg & 29 & 0.495 & $0.304-0.806$ & 0.005 & 0.998 & $0.0 \%$ \\
\hline & Sitagliptin $25 \mathrm{mg}$ & 1 & 5.240 & $0.247-111.319$ & 0.288 & 0.000 & $0.0 \%$ \\
\hline & Sitagliptin 50 mg & 1 & 0.317 & $0.013-7.841$ & 0.483 & 0.000 & $0.0 \%$ \\
\hline & Vildagliptin $100 \mathrm{mg}$ & 3 & 0.566 & $0.120-2.678$ & 0.473 & 0.455 & $0.0 \%$ \\
\hline & Vildagliptin 50 mg & 3 & 1.370 & $0.270-6.952$ & 0.704 & 0.537 & $0.0 \%$ \\
\hline
\end{tabular}

DPP-4 — dipeptidyl peptidase 4; GLP-1 — glucagon-like peptide 1 WMD — weighted mean difference; BMI — body mass index; CI — confidence interval

of whom 1265 individuals had fractures (628 individuals were in the experimental group and 637 individuals were in the control group).

The age of the included patients ranged from 49.4 to 71.6 years. The length of follow-up period ranged from 12 to 234 weeks. The sample sizes of individual trials were between 21 and 16,492 patients. Among all of the patients included, the mean $\mathrm{HbA}_{1 \mathrm{c}}$ was $7.96 \pm 1.10 \%$, mean BMI was $30.59 \pm 3.75 \mathrm{~kg} / \mathrm{m}^{2}$, and mean duration of diabetes was $7.89 \pm 3.92$ years. Fifty-nine trials were placebo-controlled, and 63 trails used an active comparator, which included canagliflozin, empagliflozin, dapagliflozin, glipizide, glimepiride, metformin, voglibose, insulin, and pioglitazone.

\section{Odds ratio of fracture}

The fixed-effects model was used on account of the $\mathrm{I}^{2}$ test for heterogeneity $\left(\mathrm{I}^{2}=0.0 \%\right)$. The pooled OR of fracture for patients treated with incretins versus controls was 0.97 (95\% CI: 0.88-1.08), which showed that incretins did not enhance the risk of fracture (Fig. 4). The pooled OR of fracture patients in DPP4-Is and GLP1-ras were 0.98 [0.87-1.10] and 0.94 [0.72-1.23], respectively (Tab. 1). Subgroup analysis was performed to explore which factors had an effect on the OR of fractures with incretins, whereas $p$ values of most of the subgroup analysis (different areas; different classification of incretins; different types of incretins; different doses of incretins; different types of control; different lengths of follow-up; mean age; mean $\mathrm{HbA}_{1 \mathrm{c}}$; mean $\mathrm{BMI}$; mean diabetes duration) were greater than 0.05 . The only significant difference was observed in the different doses of incretins when the subgroups were stratified by sitagliptin $100 \mathrm{mg}$ and liraglutide $1.8 \mathrm{mg}$ ( $\mathrm{p}=0.005$ and $\mathrm{p}=0.022$, respectively). The fractures occurred mainly in NCT00968708, NCT00295633, NCT01107886,
NCT00790205, NCT01703208, NCT01179048, and NCT01272219, as shown in Supplementary File - Table S1.

\section{Publication bias}

Based on Egger's test ( $p=0.735)$ and visual inspection, the asymmetrical funnel plot indicated no major publication bias (Fig. 5).

\section{Heterogeneity analysis}

No heterogeneity was observed among these studies $\left(\mathrm{I}^{2}=0.0 \%, \mathrm{p}=0.996\right)$. As can be seen from the Galbraith plot, compared to other trials, the heterogeneity of NCT01179048 and NCT00295633 is significant. Multiple meta-regression was performed with $p$ values of covariate (classification of incretins, type of experimental drugs, type of control drugs, experimental group dose, region, age, $\mathrm{BMI}$, intervention time, diabetes duration, glycosylated haemoglobin) were greater than 0.1 , indicated none of these factors contribute to heterogeneity. Meanwhile, subgroup analyses based on study characteristics were performed to further explore possible sources of heterogeneity. In addition to liraglutide $1.2 \mathrm{mg}$ and saxagliptin $5 \mathrm{mg}\left(\mathrm{I}^{2}=47.0 \%, \mathrm{I}^{2}=33.7 \%\right)$, other's heterogeneity is $0.0 \%$. The heterogeneity is acceptable if it is less than $50 \%$.

\section{Sensitivity analysis}

The multiple sensitivity was analysed to evaluate the robustness of results among studies. These were based on the following: (1) selection of different effect models; (2) selection of different effect sizes; (3) trim and fill method; and (4) investigating the effect of a single trial on the whole. The results showed that the selection of different effect models (random-effect model, MH-OR 0.971, 95\% CI: 0.872-1.082) and different effect sizes (MH-RR 0.973, 95\% CI: 0.878-1.077) had no obvious effect on the 


\begin{tabular}{|c|c|c|c|c|c|c|c|c|c|c|c|}
\hline \multirow[t]{2}{*}{ Study/Subgroup } & \multicolumn{2}{|c|}{ No of events/total } & \multirow{2}{*}{$\begin{array}{c}\text { MH fixed OR } \\
(95 \% \mathrm{CI})\end{array}$} & \multirow{2}{*}{$\begin{array}{l}\text { Weight } \\
(\%)\end{array}$} & \multirow{2}{*}{$\begin{array}{c}\text { MH fixed OR } \\
(95 \% \mathrm{Cl})\end{array}$} & \multirow[t]{2}{*}{ Study/Subgroup } & \multicolumn{2}{|c|}{ No of events/total } & \multirow{2}{*}{$\begin{array}{c}\text { MH fixed OR } \\
(95 \% \mathrm{Cl})\end{array}$} & \multirow{2}{*}{$\begin{array}{c}\text { Weight } \\
(\%)\end{array}$} & \multirow{2}{*}{$\begin{array}{c}\text { MH fixed OR } \\
(95 \% \mathrm{CI})\end{array}$} \\
\hline & Incretin & Control & & & & & Incretin & Control Control & & & \\
\hline Bosi 2009 & $1 / 292$ & $0 / 292$ & & 0.07 & $3.01(0.12$ to 74.20$)$ & NCT00838916 & $2 / 504$ & $0 / 241$ & & 0.09 & $2.40(0.12$ to 50.25$)$ \\
\hline Iwamoto(a) 2010 & $0 / 163$ & $1 / 156$ & & 0.21 & 0.32 (0.01 to 7.84$)$ & NCT00849017 & $1 / 101$ & $0 / 101$ & & 0.07 & $3.03(0.12$ to 75.26$)$ \\
\hline Iwamoto(b) 2010 & $0 / 188$ & $2 / 192$ & & 0.34 & 0.20 (0.01 to 4.24$)$ & NCT00856284(a) & $3 / 885$ & $4 / 874$ & & 0.56 & $0.74(0.17$ to 3.32$)$ \\
\hline NCT00082381 & $0 / 282$ & $1 / 267$ & & 0.21 & 0.31 (0.01 to 7.75$)$ & NCT00856284(b) & $3 / 880$ & $4 / 874$ & & 0.56 & $0.74(0.17$ to 3.33$)$ \\
\hline NCT00082407 & $1 / 253$ & $0 / 248$ & & 0.07 & $2.95(0.12$ to 72.82$)$ & NCT00866 658 & $1 / 154$ & $1 / 157$ & 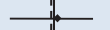 & 0.14 & $1.02(0.06$ to 16.45$)$ \\
\hline NCT00086502 & $0 / 175$ & $1 / 178$ & & 0.21 & 0.34 (0.01 to 8.33$)$ & NCT00881530 & $0 / 56$ & $1 / 56$ & - & 0.21 & 0.33 (0.01 to 8.21$)$ \\
\hline NCT00086515 & $0 / 464$ & $1 / 237$ & & 0.28 & $0.17(0.01$ to 4.18$)$ & NCT00885352 & $0 / 157$ & $1 / 156$ & - & 0.21 & $0.33(0.01$ to 8.14$)$ \\
\hline NCT000 87516 & $0 / 238$ & $1 / 130$ & & 0.27 & 0.18 (0.01 to 4.48$)$ & NCT00915772 & $1 / 225$ & $1 / 170$ & - & 0.16 & $0.75(0.05$ to 12.15$)$ \\
\hline NCT00094770 & $3 / 588$ & $3 / 584$ & & 0.42 & 0.99 (0.2 to 4.94$)$ & NCT00935532 & $2 / 215$ & $0 / 212$ & & 0.07 & 4.98 (0.24 to 104.28$)$ \\
\hline NCT00099931 & $0 / 144$ & $1 / 152$ & & 0.20 & 0.35 (0.01 to 8.65$)$ & NCT00954447 & $5 / 631$ & $6 / 630$ & - & 0.83 & $0.83(0.25$ to 2.74$)$ \\
\hline NCT00101712 & $0 / 156$ & $1 / 150$ & & 0.21 & 0.32 (0.01 to 7.88$)$ & NCT00960661 & $2 / 315$ & $0 / 312$ & & 0.07 & 4.98 (0.24 to 104.24$)$ \\
\hline NCT00103857(a) & $0 / 179$ & $1 / 182$ & & 0.21 & 0.34 (0.01 to 8.33$)$ & NCT00968708 & $38 / 2676$ & $50 / 2698$ & & 6.85 & $0.76(0.5$ to 1.17$)$ \\
\hline NCT00103857(b) & $0 / 179$ & $2 / 182$ & & 0.34 & 0.20 (0.01 to 4.22$)$ & NCT01006603 & $9 / 359$ & $4 / 359$ & & 0.54 & $2.28(0.7$ to 7.48$)$ \\
\hline NCT00121667(a) & $1 / 181$ & $0 / 179$ & & 0.07 & $2.98(0.12$ to 73.72$)$ & NCT01012037 & $3 / 447$ & $0 / 44$ & & 0.13 & $0.70(0.04$ to 13.79$)$ \\
\hline NCT00121667(b) & $1 / 191$ & $0 / 179$ & & 0.07 & $2.83(0.11$ to 69.84$)$ & NCT01023581 & $0 / 445$ & $1 / 334$ & - & 0.24 & 0.25 (0.01 to 6.15$)$ \\
\hline NCT00121667(c) & $2 / 192$ & $0 / 179$ & & 0.07 & $4.71(0.23$ to 98.81$)$ & NCT01075282(a) & $1 / 273$ & $0 / 262$ & & 0.07 & $2.89(0.12$ to 71.26$)$ \\
\hline NCT00286468 & $1 / 198$ & $0 / 99$ & & 0.09 & $1.51(0.06$ to 37.44$)$ & NCT010 75282(b) & $1 / 272$ & $0 / 262$ & & 0.07 & $2.90(0.12$ to 71.52$)$ \\
\hline NCT00294723(a) & $2 / 251$ & $1 / 248$ & & 0.14 & $1.98(0.18$ to 22.02$)$ & NCT01076075 & $0 / 210$ & $1 / 212$ & - & 0.21 & 0.34 (0.01 to 8.27$)$ \\
\hline NCT00294723(b) & $0 / 246$ & $1 / 248$ & & 0.21 & 0.34 (0.01 to 8.26$)$ & NCT01076088 & $0 / 247$ & $3 / 250$ & & 0.48 & 0.14 (0.01 to 2.78$)$ \\
\hline NCT00295633(a) & $4 / 195$ & $2 / 184$ & & 0.28 & $1.91(0.35$ to 10.53$)$ & NCT01084005 & $2 / 162$ & 0/79 & & 0.09 & $2.48(0.12$ to 52.2$)$ \\
\hline NCT00295633(b) & $1 / 186$ & $11 / 184$ & & 1.53 & 0.09 (0.01 to 0.67$)$ & NCT01 106677 & $0 / 368$ & $2 / 366$ & & 0.35 & 0.20 (0.01 to 4.14$)$ \\
\hline NCT00305604 & $0 / 102$ & $2 / 104$ & & 0.34 & 0.20 (0.01 to 4.22$)$ & NCT01 106690 & $0 / 115$ & $1 / 113$ & & 0.21 & 0.33 (0.01 to 8.05$)$ \\
\hline NCT00313313 & $1 / 248$ & $0 / 267$ & & 0.07 & $3.24(0.13$ to 79.97$)$ & NCT01107886 & $241 / 8280$ & $240 / 8212$ & - & 32.63 & 1.00 (0.83 to 1.19$)$ \\
\hline NCT00327015 & $3 / 643$ & $0 / 328$ & & 0.09 & $3.59(0.19$ to 69.71$)$ & NCT01126580 & $1 / 269$ & $0 / 268$ & & 0.07 & $3.00(0.12$ to 73.97$)$ \\
\hline NCT00328627 & $0 / 129$ & $1 / 129$ & & 0.21 & $0.33(0.01$ to 8.2$)$ & NCT01137812 & $1 / 378$ & $2 / 377$ & & 0.28 & $0.50(0.05$ to 5.51$)$ \\
\hline NCT00337610 & 0/96 & $1 / 94$ & & 0.21 & 0.32 (0.01 to 8.03$)$ & NCT01177813 & $0 / 223$ & $1 / 224$ & & 0.21 & $0.33(0.01$ to 8.23$)$ \\
\hline NCT0 0350779 & $0 / 170$ & $1 / 92$ & & 0.27 & 0.18 (0.01 to 4.44$)$ & NCT01 179048 & $35 / 4668$ & $58 / 4672$ & & 8.02 & $0.60(0.39$ to 0.92$)$ \\
\hline NCT00359762 & $7 / 508$ & $5 / 511$ & & 0.69 & $1.41(0.45$ to 4.49$)$ & NCT01 183013 & $1 / 133$ & $0 / 140$ & & 0.07 & $3.18(0.13$ to 78.78$)$ \\
\hline NCT00372060 & $0 / 66$ & $1 / 68$ & & 0.20 & $0.34(0.01$ to 8.46$)$ & NCT01 191268(a) & $2 / 295$ & $3 / 296$ & - & 0.41 & $0.67(0.11$ to 4.02$)$ \\
\hline NCT00395343 & $1 / 322$ & $0 / 319$ & & 0.07 & $2.98(0.12$ to 73.46$)$ & NCT01 191268(b) & $3 / 293$ & $3 / 296$ & & 0.41 & 1.01 (0.20 to 5.05$)$ \\
\hline NCT00397631 & $1 / 261$ & $0 / 259$ & & 0.07 & 2.99 (0.12 to 73.7$)$ & NCT01204294 & $1 / 228$ & $0 / 124$ & & 0.09 & $1.64(0.07$ to 40.60$)$ \\
\hline NCT00411554 & $0 / 155$ & $1 / 147$ & & 0.21 & 0.31 (0.01 to 7.77$)$ & NCT01215097 & $1 / 205$ & $0 / 100$ & & 0.09 & $1.47(0.06$ to 36.51$)$ \\
\hline NCT00432276 & 6/404 & $4 / 399$ & & 0.55 & $1.49(0.42$ to 5.32$)$ & NCT01272219(a) & $4 / 957$ & $0 / 487$ & & 0.09 & $4.6(0.25$ to 85.64$)$ \\
\hline NCT00434954 & $3 / 247$ & $0 / 233$ & & 0.07 & $6.69(0.34$ to 130.12$)$ & NCT01272219(b) & $8 / 1524$ & $1 / 755$ & & 0.19 & $3.98(0.5$ to 31.87$)$ \\
\hline NCT00449930 & $1 / 455$ & $0 / 439$ & & 0.07 & $2.90(0.12$ to 71.4$)$ & NCT01272232 & $1 / 210$ & $0 / 212$ & & 0.07 & 3.04 (0.12 to 75.12$)$ \\
\hline NCT00482729 & $1 / 625$ & $2 / 621$ & & 0.28 & $0.50(0.05$ to 5.48$)$ & NCT01289990 & $0 / 223$ & $2 / 168$ & - & 0.40 & $0.15(0.01$ to 3.12$)$ \\
\hline NCT00509236 & $2 / 64$ & $0 / 65$ & & 0.07 & $5.24(0.25$ to 111.32$)$ & NCT01296412 & $0 / 324$ & $3 / 326$ & _ & 0.49 & 0.14 (0.01 to 2.77$)$ \\
\hline NCT00509262 & $1 / 211$ & $1 / 212$ & & 0.14 & $1.01(0.06$ to 16.17$)$ & NCT01388361 & $0 / 87$ & $1 / 86$ & & 0.21 & 0.33 (0.01 to 8.11$)$ \\
\hline NCT00516074 & $1 / 28$ & $0 / 26$ & & 0.07 & $2.89(0.11$ to 74.17$)$ & NCT01422876 & $0 / 267$ & $1 / 276$ & - & 0.21 & 0.34 (0.01 to 8.47$)$ \\
\hline NCT00575588 & $4 / 428$ & $2 / 430$ & & 0.28 & $2.02(0.37$ to 11.08$)$ & NCT01438814 & $0 / 344$ & $1 / 345$ & - & 0.21 & 0.33 (0.01 to 8.21$)$ \\
\hline NCT00601250 & $1 / 523$ & $0 / 177$ & & 0.10 & $1.02(0.04$ to 25.13$)$ & NCT01462266 & $0 / 329$ & $1 / 329$ & & 0.21 & $0.33(0.01$ to 8.19$)$ \\
\hline NCT00602472 & 3/792 & $0 / 263$ & & 0.10 & $2.34(0.12$ to 45.38$)$ & NCT01512108 & $0 / 240$ & $1 / 120$ & - & 0.28 & $0.17(0.01$ to 4.10$)$ \\
\hline NCT00614939 & $0 / 85$ & $1 / 85$ & & 0.21 & 0.33 (0.01 to 8.20$)$ & NCT01557166 & $1 / 176$ & $0 / 179$ & & 0.07 & $3.07(0.12$ to 75.83$)$ \\
\hline NCT00621140 & $1 / 336$ & $2 / 167$ & & 0.37 & $0.25(0.02$ to 2.74$)$ & NCT01584232 & $1 / 181$ & $0 / 180$ & & 0.07 & $3.00(0.12$ to 74.13$)$ \\
\hline NCT00622284 & 9/776 & $4 / 775$ & & 0.55 & $2.26(0.69$ to 7.38$)$ & NCT01606007 & $1 / 176$ & $0 / 179$ & & 0.07 & $3.07(0.12$ to 75.83$)$ \\
\hline NCT00661362 & $3 / 283$ & $0 / 287$ & & 0.07 & $7.18(0.37$ to 139.53$)$ & NCT01617434 & $1 / 225$ & $1 / 225$ & & 0.14 & $1.00(0.06$ to 16.09$)$ \\
\hline NCT00688701 & $0 / 239$ & $1 / 122$ & & 0.28 & $0.17(0.01$ to 4.18$)$ & NCT01621178(a) & $0 / 190$ & $3 / 194$ & - & 0.48 & $0.14(0.01$ to 2.80$)$ \\
\hline NCT00701090 & $2 / 516$ & $1 / 519$ & & 0.14 & $2.02(0.18$ to 22.30$)$ & NCT01621178(b) & $0 / 192$ & $3 / 194$ & - & 0.48 & 0.14 (0.01 to 2.77$)$ \\
\hline NCT00707993 & $2 / 222$ & $1 / 219$ & & 0.14 & $1.98(0.18$ to 22.02$)$ & NCT01697592 & $2 / 191$ & $0 / 196$ & & 0.07 & $5.19(0.25$ to 108.7$)$ \\
\hline NCT00712673 & $5 / 510$ & $0 / 170$ & & 0.10 & $3.71(0.2$ to 67.45$)$ & NCT01698775 & $1 / 106$ & $0 / 106$ & & 0.07 & $3.03(0.12$ to 75.19$)$ \\
\hline NCT0 0713830 & $4 / 574$ & $1 / 285$ & & 0.19 & $1.99(0.22$ to 17.91$)$ & NCT01703208 & $17 / 2100$ & $13 / 2102$ & $\leftarrow$ & 1.80 & 1.31 (0.64 to 2.71$)$ \\
\hline NCT00715624 & $2 / 328$ & $1 / 167$ & & 0.18 & $1.02(0.09$ to 11.31$)$ & NCT01769378 & $1 / 239$ & $0 / 60$ & & 0.11 & $0.76(0.03$ to 18.91$)$ \\
\hline NCT00722371(a) & $1 / 231$ & $1 / 230$ & & 0.14 & $1.00(0.06$ to 16.01$)$ & NCT01792518 & $1 / 182$ & $0 / 178$ & & 0.07 & $2.95(0.12$ to 72.91$)$ \\
\hline NCT00722371(b) & $1 / 231$ & $1 / 233$ & & 0.14 & $1.01(0.06$ to 16.22$)$ & NCT01798706 & $2 / 176$ & $1 / 174$ & & 0.14 & $1.99(0.18$ to 22.13$)$ \\
\hline NCT00722371(c) & $1 / 231$ & $0 / 230$ & & 0.07 & $3.00(0.12$ to 74.03$)$ & NCT02002221 & $1 / 78$ & $0 / 78$ & & 0.07 & $3.04(0.12$ to 75.75$)$ \\
\hline NCT00757588 & $2 / 304$ & $3 / 151$ & & 0.56 & $0.33(0.05$ to 1.98$)$ & NCT02058147 & $0 / 233$ & $1 / 467$ & & 0.14 & $0.67(0.03$ to 16.41$)$ \\
\hline NCT00763451 & $3 / 322$ & $2 / 160$ & & 0.37 & $0.74(0.12$ to 4.49$)$ & NCT02104804 & $1 / 234$ & $2 / 231$ & & 0.28 & $0.49(0.04$ to 5.46$)$ \\
\hline NCT00765817 & $0 / 137$ & $1 / 122$ & & 0.22 & 0.30 (0.01 to 7.30$)$ & NCT02152371 & $4 / 150$ & $0 / 150$ & 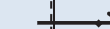 & 0.07 & 9.25 (0.49 to 173.25$)$ \\
\hline NCT00790205 & $189 / 7332$ & 186/7339 & & 25.26 & 1.02 (0.83 to 1.25$)$ & NCT02229383 & $1 / 232$ & $0 / 231$ & 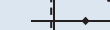 & 0.07 & $3.00(0.12$ to 74.03$)$ \\
\hline NCT00798161 & $1 / 143$ & $1 / 147$ & & 0.14 & $1.03(0.06$ to 16.6$)$ & Pan 2012 & $1 / 148$ & $0 / 144$ & & 0.07 & $2.94(0.12$ to 72.74$)$ \\
\hline NCT00800683 & $2 / 68$ & $0 / 65$ & & 0.07 & $4.93(0.23$ to 104.56$)$ & Yang 2015 & $3 / 37$ & $0 / 40$ & & 0.06 & $8.22(0.41$ to 164.68$)$ \\
\hline Total $(95 \% \mathrm{Cl})$ & $628 / 58706$ & $637 / 52833$ & & 100.00 & & & $0.97(0.88$ to 1 . & & & & \\
\hline & & & $\begin{array}{lll}0.1 & 1 & 100\end{array}$ & & & & & & & & \\
\hline & & Favours & incretins & rs control & & & & Favour & incretins & rs control & \\
\hline & & & & & Heterogeneity: $\chi^{2}$ & $97, \mathrm{df}=121, \mathrm{p}=$ & $=0.0 \%$ & & & & \\
\hline
\end{tabular}

Figure 4. Meta-analysis of the use of incretins and fracture risk compared to control

conclusion, and the results were stable. Five trials with unclear risk of bias were excluded, and the combined effect altered from (OR: $-0.029,95 \%$ CI: $-0.137-0.079)$ to (OR: -0.042 , 95\% CI: $-0.150-0.065)$. There was no 

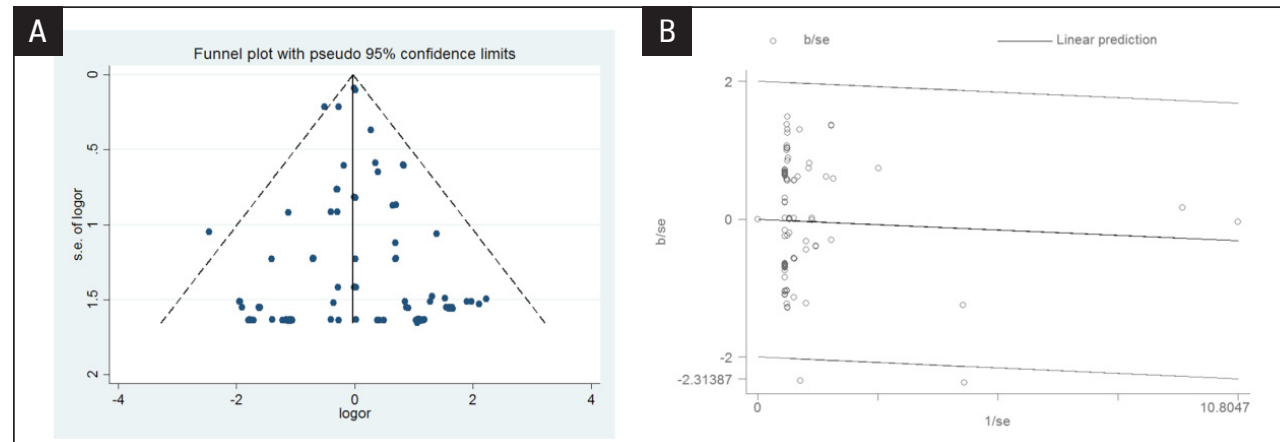

C

D
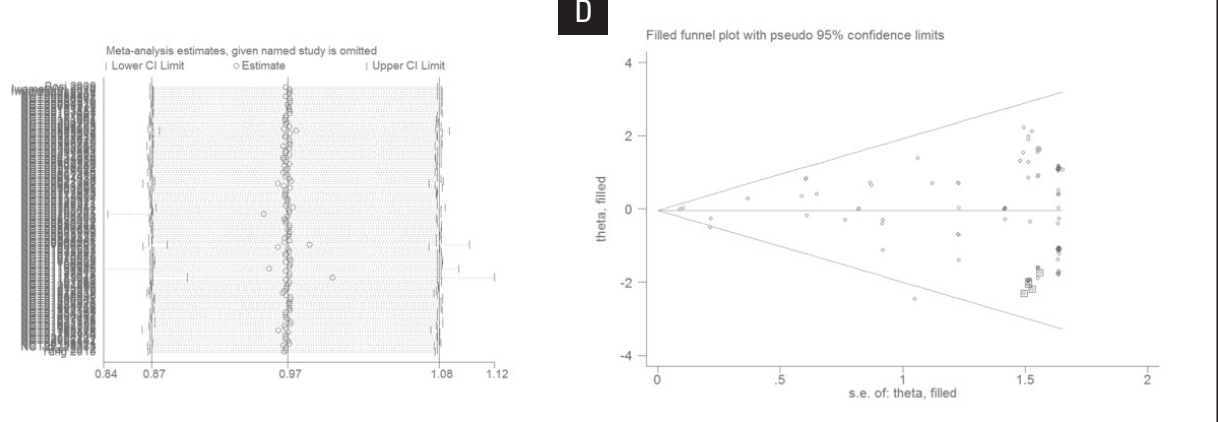

Figure 5. The results of funnel plots (A), Galbraith plot (B), sensitivity analysis and trim $(\boldsymbol{C})$, and fill plot (D) of the use of incretins and fracture risk compared to control

significant change before and after the pruning, and the results were stable. In addition, the effect of a single trial on the combined effect was investigated. It was found that a single study has little effect on the combined effect.

\section{Quality of evidence}

The quality assessment of the 122 trials is summarized in Figure 2-3. Among these trials, 70 (57.4\%) adequately generated their randomization sequence, 97 (79.5\%) concealed allocation, $101(82.8 \%)$ blinded participants and personnel, $62(50.8 \%$ ) blinded outcome assessment, and $111(92.5 \%)$ reported complete outcome data.

\section{Discussion}

Our meta-analysis showed that the use of incretins did not enhance the fracture risk (OR: 0.972, 95\% CI: 0.876-1.079) compared with placebo or other active drugs. Also, in subgroup analysis, we found that the dose administered in groups of sitagliptin $100 \mathrm{mg}$ (OR: 0.495, 95\% CI: 0.304-0.806) and liraglutide $1.8 \mathrm{mg}$ (OR: $0.621,95 \%$ CI: $0.413-0.933)$ can reduce the risk of fracture. No other subgroups were found to either reduce or increase the risk of fracture.

In subgroup analysis, it was not the first discovery that the dose administered in groups of liraglutide $1.8 \mathrm{mg}$ (OR: $0.621,95 \%$ CI: 0.413-0.933) can reduce the risk of fracture $[22,23]$. However, we still know little about the interpretation of how to decrease fracture risk among T2DM patients during subsequent treat- ment with incretins. We know that GLP-1 can reduce the risk of fracture, mainly related to increasing bone mass, improving trabecular and cortical architectures, enhancing bone strength and tissue material properties, and affecting the collagen compartment rather than the mineral one. Moreover, the effect of liraglutide on bone may be increased by osteoblastic markers such as Runx2, -1 coll, and ALP [11, 24]. Some research showed that liraglutide is widely used all over the world, and it shares $97 \%$ sequence identity with native GLP-1 [25, 26]. Therefore, we hypothesized that the probable reason for liraglutide reducing the fracture risk is similar to that for GLP-1.

We have confirmed for the first time that sitagliptin $100 \mathrm{mg}$ (OR: 0.495, 95\% CI: 0.304-0.806) has a protective effect on fracture by meta-analysis. Sitagliptin is the first DPP-4 inhibitor to be used in clinical practice. Cusick et al. [27] proved in diabetic rats that sitagliptin probably reduced the bone resorption marker cyclophosphamide and attenuated bone loss to increase bone strength independently of the hypoglycaemic effect. Similarly, in clinical studies, Hegazy et al. [28] showed that after 12 weeks, compared with metformin treatment, sitagliptin treatment led to a slight increase in bone transformation markers and bone mineral density in the vertebrae. This may partly explain why sitagliptin $100 \mathrm{mg}$ showed positive effects in our meta-analysis, with a reduced risk of fracture.

Although the differences of other subgroup analyses (mean BMI; different lengths of follow-up; differ- 
ent areas; different types of control; mean age; mean $\mathrm{HbA}_{1 c^{\prime}}$ mean diabetes duration) were not statistically significant, it was meaningful to conduct subgroup analysis. For example, it is well known that BMI is positively correlated with bone mineral density and negatively correlated with the incidence of osteoporosis, which is a protective factor for bone mineral density [29]. Therefore, the effect of drugs on patients' BMI may have different effects on fracture outcome. Incretin users are generally overweight, and weight loss-induced bone mass decreases after using incretins, both of which may weaken the positive effect of incretins on bone metabolism, resulting in a neutral result of the fracture risk after incretin treatment in clinical trials. The fracture risk in thiazolidinediones (TZD) users was not exposed in the preclinical study, registration, and listing research. The risk of fractures was revealed after the five-year follow-up [30]. Different follow-up times may affect the outcome of the study, so we conducted subgroup analysis on the follow-up time. Although we did not draw conclusions on the effect of follow-up time of incretins on fractures, we can explore the association between incretin use and fracture risk during long duration incretin therapy.

Although $\mathrm{HbA}_{1 \mathrm{c}}$ had no significant difference in our subgroup analysis results, according to relevant studies, excessive blood sugar can form glycation end products (AGEs) with proteins, amino acids, peptides, and nucleic acids in the body. AGE accumulation can reduce bone strength, inhibit the expression of osteoblast phenotype, and promote osteoblast apoptosis, which leads to insufficient bone formation [24]. Thus, we still believe that positively alleviating the toxicity of high glucose and relieving the damage of bones caused by high glucose toxicity is beneficial to bone metabolism.

The advantages of this article are the first inclusion of RCT research data in cohort studies and that GLP1-ras and DPP4-Is are used as research objects for meta-analysis. Secondly, through a scientific and rigorous retrieval process as well as careful screening, our meta-analysis sample size was the largest, involving a total of 122 trials studies with 40 studies for GLP1-ras and 82 studies for DPP4-Is including 111,539 patients. Furthermore, it was the most comprehensive meta-analysis of the data, and we conducted multilevel subgroup analysis. Finally, our results not only proved to be robust through a variety of sensitivity analysis methods, but also no major publication and no heterogeneity was observed among these studies.

The disadvantages of this article are that the follow-up time in these studies is relatively short (the length of follow-up period ranged from 12 to 234 weeks). As TZD drugs, the risk of fractures was revealed after the five-year follow-up. It is suggested that the risk of fracture of the drug may be confirmed by long-term clinical data. In addition, the included studies lacked a record of the subjects' lifestyle, physical activity, smoking, drinking, etc., and if there are differences in these factors in the experimental group and control group, it may lead to biased results regarding the effect on fracture [31]. Moreover, the included studies were mostly based on $\mathrm{HbA}_{1 \mathrm{c}}$ and cardiovascular events as primary outcome measures, but fracture events were ignored and not reported as adverse events which may be one of the reasons why our results are negative.

The above reason may cause including studies only partially reflected the occurrence of the drug-induced fracture events [32]. But in TECOS, fractures occurred at a rate of 9/1000 per year [33]. Hence, the skeletal safety profiles of diabetic medications deserve attention, and further investigation is necessary. Considering the limitations of existing RCT trials, we suggest considering the following four aspects. Firstly, fracture events should be taken as the main study endpoint. In order to ensure patient level consistency, the recording of patients' basic information is necessary. In addition, fracture evaluation methods should include the bone mineral density and bone metabolism indicators, etc. What is more, sufficiently long follow-up events are also essential, which can clearly explain the association.

\section{Conclusions}

This meta-analysis suggested that current use of incretins does not increase the risk of fracture in type 2 diabetes patients from RCT studies. It also showed a protective effect on bone metabolism when sitagliptin $100 \mathrm{mg}$ or liraglutide $1.8 \mathrm{mg} /$ day was administrated. Therefore, we believe that the use of incretins does not increase the risk of fracture when used in patients with T2DM. In addition, we consider that older patients are prone to osteoporosis, so we suggest that these people be treated with hypoglycaemic therapy with drugs such as sitagliptin or liraglutide, which may have bone-protective effects.

\section{Declaration of interest}

The authors declare no conflicts of interest.

\section{Funding}

This research was supported by the Natural Science Research Projects of Jiangsu Colleges and Universities (17KJB350002).

\section{Acknowledgement}

The authors thank Daphne Liao, Pharm.D and Lei Zou, Pharm.D, of MCPHS University for linguistic review of the manuscript. 


\section{References}

1. Braithwaite RS, Col NF, Wong JB. Estimating hip fracture morbidity, mortality and costs. J Am Geriatr Soc. 2003; 51(3): 364-370, doi: 10.1046/ j.1532-5415.2003.51110.x indexed in Pubmed: 12588580.

2. Moayeri A, Mohamadpour M, Mousavi SF, et al. Fracture risk in patients with type 2 diabetes mellitus and possible risk factors: a systematic review and meta-analysis. Ther Clin Risk Manag. 2017; 13: 455-468, doi: 10.2147/TCRM.S131945, indexed in Pubmed: 28442913.

3. Wolverton D, Blair MM. Fracture risk associated with common medications used in treating type 2 diabetes mellitus. Am J Health Syst Pharm. 2017; 74(15): 1143-1151, doi: 10.2146/ajhp160319, indexed in Pubmed: 28743778.

4. Schwartz AV, Lecka-Czernik B. Diabetes, bone and glucose-lowering agents: basic biology. Diabetologia. 2017; 60(7): 1163-1169, doi: 10.1007/s00125-017-4269-4, indexed in Pubmed: 28434032.

5. Schwartz AV, Lecka-Czernik B. Diabetes, bone and glucose-lowering agents: basic biology. Diabetologia. 2017; 60(7): 1163-1169, doi: 10.1007/s00125-017-4269-4, indexed in Pubmed: 28434032.

6. Schott G, Martinez YV, Ediriweera de Silva RE, et al. Effectiveness and safety of dipeptidyl peptidase 4 inhibitors in the management of type 2 diabetes in older adults: a systematic review and development of recommendations to reduce inappropriate prescribing. BMC Geriatr. 2017; 17(Suppl 1): 226, doi: 10.1186/s12877-017-0571-8, indexed in Pubmed: 29047372.

7. Tasyurek HM, Altunbas HA, Balci MK, et al. Incretins: their physiology and application in the treatment of diabetes mellitus. Diabetes Metab Res Rev. 2014; 30(5): 354-371, doi: 10.1002/dmrr.2501, indexed in Pubmed: 24989141.

8. Nauck MA, Meier JJ. Incretin hormones: Their role in health and disease. Diabetes Obes Metab. 2018; 20 Suppl 1: 5-21, doi: 10.1111/dom.13129, indexed in Pubmed: 29364588.

9. Monami M, Dicembrini I, Antenore A, et al. Dipeptidyl peptidase-4 inhibitors and bone fractures: a meta-analysis of randomized clinical trials. Diabetes Care. 2011; 34(11): 2474-2476, doi: 10.2337/dc11-1099, indexed in Pubmed: 22025784.

10. Mabilleau G, Mieczkowska A, Chappard D. Use of glucagon-like peptide- 1 receptor agonists and bone fractures: a meta-analysis of randomized clinical trials. J Diabetes. 2014; 6(3): 260-266, doi: 10.1111/1753-0407.12102, indexed in Pubmed: 24164867.

11. Su B, Sheng H, Zhang M, et al. Risk of bone fractures associated with glucagon-like peptide-1 receptor agonists' treatment: a meta-analysis of randomized controlled trials. Endocrine. 2015; 48(1): 107-115, doi: 10.1007/s12020-014-0361-4, indexed in Pubmed: 25074632.

12. Fu J, Zhu J, Hao Y, et al. Dipeptidyl peptidase-4 inhibitors and fracture risk: an updated meta-analysis of randomized clinical trials. Sci Rep. 2016; 6: 29104, doi: 10.1038/srep29104, indexed in Pubmed: 27384445.

13. Dombrowski S, Kostev K, Jacob L. Use of dipeptidyl peptidase-4 inhibitors and risk of bone fracture in patients with type 2 diabetes in Germany-A retrospective analysis of real-world data. Osteoporos Int. 2017; 28(8): 2421-2428, doi: 10.1007/s00198-017-4051-y, indexed in Pubmed: 28455750.

14. Yang J, Huang C, Wu S, et al. The effects of dipeptidyl peptidase-4 inhibitors on bone fracture among patients with type 2 diabetes mellitus: A network meta-analysis of randomized controlled trials. PLoS One. 2017; 12(12): e0187537, doi: 10.1371/journal.pone.0187537, indexed in Pubmed: 29206832.

15. Driessen JHM, de Vries $\mathrm{F}$, van Onzenoort $\mathrm{H}$, et al. The use of incretins and fractures - a meta-analysis on population-based real life data. $\mathrm{Br}$ J Clin Pharmacol. 2017; 83(4): 923-926, doi: 10.1111/bcp.13167, indexed in Pubmed: 27780288.

16. Knobloch K, Yoon U, Vogt PM. Preferred reporting items for systematic reviews and meta-analyses (PRISMA) statement and publica- tion bias. J Craniomaxillofac Surg. 2011; 39(2): 91-92, doi: 10.1016/j. jcms.2010.11.001, indexed in Pubmed: 21145753.

17. Higgins JPT, Thompson SG, Deeks JJ, et al. Measuring inconsistency in meta-analyses. BMJ. 2003; 327(7414): 557-560, doi: 10.1136/bmj.327.7414.557, indexed in Pubmed: 12958120.

18. Jadad AR, Moore RA, Carroll D, et al. Assessing the quality of reports of randomized clinical trials: is blinding necessary? Control Clin Trials. 1996; 17(1): 1-12, doi: 10.1016/0197-2456(95)00134-4, indexed in Pubmed: 8721797.

19. Cumpston M, Li T, Page MJ, et al. Updated guidance for trusted systematic reviews: a new edition of the Cochrane Handbook for Systematic Reviews of Interventions. Cochrane Database Syst Rev. 2019; 10: ED000142, doi: 10.1002/14651858.ED000142, indexed in Pubmed: 31643080.

20. Begg C, Mazumdar M. Operating Characteristics of a Rank Correlation Test for Publication Bias. Biometrics. 1994; 50(4): 1088, doi: 10.2307/2533446, indexed in Pubmed: 7786990.

21. Egger M, Davey Smith G, Schneider M, et al. Bias in meta-analysis detected by a simple, graphical test. BMJ. 1997; 315(7109): 629-634, doi: 10.1136/bmj.315.7109.629, indexed in Pubmed: 9310563.

22. Gilbert MP, Marre M, Holst JJ, et al. Comparison of the Long-Term Effects of Liraglutide and Glimepiride Monotherapy on Bone Mineral Density in Patients with Type 2 Diabetes. Endocr Pract. 2016; 22(4): 406-411, doi: 10.4158/EP15758.OR, indexed in Pubmed: 26574791.

23. Wen $\mathrm{B}, \mathrm{Zhao} \mathrm{Lu}$, Zhao $\mathrm{H}$, et al. Liraglutide exerts a bone-protective effect in ovariectomized rats with streptozotocin-induced diabetes by inhibiting osteoclastogenesis. Exp Ther Med. 2018; 15(6): 5077-5083, doi: 10.3892/etm.2018.6043, indexed in Pubmed: 29805533

24. Mabilleau G, Pereira M, Chenu C. Novel skeletal effects of glucagon-like peptide-1 (GLP-1) receptor agonists. J Endocrinol. 2018; 236(1): R29-R42, doi: 10.1530/JOE-17-0278, indexed in Pubmed: 28855317.

25. Zhang L, Yang M, Ren $\mathrm{H}$, et al. GLP-1 analogue prevents NAFLD in ApoE KO mice with diet and Acrp30 knockdown by inhibiting c-JNK. Liver Int. 2013; 33(5): 794-804, doi: 10.1111/liv.12120, indexed in Pubmed: 23432843.

26. Vilsboll T. Liraglutide: a new treatment for type 2 diabetes. Drugs Today (Barc). 2009; 45(2): 101-113, doi: 10.1358/dot.2009.45.2.1336104, indexed in Pubmed: 19343230.

27. Cusick T, Mu J, Pennypacker BL, et al. Bone loss in the oestrogen-depleted rat is not exacerbated by sitagliptin, either alone or in combination with a thiazolidinedione. Diabetes Obes Metab. 2013; 15(10): 954-957, doi: 10.1111/dom.12109, indexed in Pubmed: 23551951.

28. Hegazy SK. Evaluation of the anti-osteoporotic effects of metformin and sitagliptin in postmenopausal diabetic women. J Bone Miner Metab. 2015; 33(2): 207-212, doi: 10.1007/s00774-014-0581-y, indexed in Pubmed: 24633493

29. Mabilleau G, Mieczkowska A, Irwin N, et al. Optimal bone mechanical and material properties require a functional glucagon-like peptide-1 receptor. J Endocrinol. 2013; 219(1): 59-68, doi: 10.1530/JOE-13-0146, indexed in Pubmed: 23911987.

30. Montagnani A, Gonnelli S, Alessandri M, et al. Osteoporosis and risk of fracture in patients with diabetes: an update. Aging Clin Exp Res. 2011; 23(2): 84-90, doi: 10.1007/BF03351073, indexed in Pubmed: 21743287.

31. Mabilleau G, Gobron B, Bouvard B, et al. Incretin-based therapy for the treatment of bone fragility in diabetes mellitus. Peptides. 2018; 100: 108-113, doi: 10.1016/j.peptides.2017.12.008, indexed in Pubmed: 29412811.

32. Scheen AJ. The safety of gliptins : updated data in 2018. Expert Opin Drug Saf. 2018; 17(4): 387-405, doi: 10.1080/14740338.2018.1444027, indexed in Pubmed: 29468916.

33. Josse RG, Majumdar SR, Zheng Y, et al. TECOS Study Group. Sitagliptin and risk of fractures in type 2 diabetes: Results from the TECOS trial. Diabetes Obes Metab. 2017; 19(1): 78-86, doi: 10.1111/dom.12786, indexed in Pubmed: 27607571. 